\title{
碱化插层二维过渡金属碳化物的制备及 其对铀酰离子的电化学检测
}

\author{
樊 惁 ${ }^{1}$, 王 琳 $^{2}$, 裴承新 ${ }^{1}$, 石伟群 ${ }^{2}$ \\ (1. 国民核生化灾害防护国家重点实验室，北京 102205; 2. 中国科学院 高能物理研究所，北京 100049)
}

摘 要: 二维过渡金属碳化物(MXenes)具有良好的电化学性能与辐照稳定性, 其在放射性核素电化学检测领域有潜 在应用价值。本研究通过碱活化的方式处理碳化钛型 MXene $\left(\mathrm{Ti}_{3} \mathrm{C}_{2} \mathrm{~T}_{x}\right)$, 随后将钾插层的 $\mathrm{Ti}_{3} \mathrm{C}_{2} \mathrm{~T}_{x}\left(\mathrm{~K}-\mathrm{Ti}_{3} \mathrm{C}_{2} \mathrm{~T}_{x}\right)$ 负载到 玻碳电极 $(\mathrm{GCE})$ 上得到 $\mathrm{K}-\mathrm{Ti}_{3} \mathrm{C}_{2} \mathrm{~T}_{x} / \mathrm{GCE}$ 修饰电极。采用 XRD、SEM、XPS 等手段分别对 $\mathrm{Ti}_{3} \mathrm{C}_{2} \mathrm{~T}_{x}$ 和 $\mathrm{K}-\mathrm{Ti}_{3} \mathrm{C}_{2} \mathrm{~T}_{x}$ 进行 分析表征, 并进一步研究了 $\mathrm{K}-\mathrm{Ti}_{3} \mathrm{C}_{2} \mathrm{~T}_{x} / \mathrm{GCE}$ 对痕量铀酰离子 $\left(\mathrm{UO}_{2}{ }^{2+}\right)$ 的电化学检测性能。循环伏安 $(\mathrm{CV})$ 实验结果表 明, 相比于 $\mathrm{GCE}$ 电极, $\mathrm{K}-\mathrm{Ti}_{3} \mathrm{C}_{2} \mathrm{~T}_{x} / \mathrm{GCE}$ 修饰电极对 $\mathrm{UO}_{2}{ }^{2+}$ 的电化学响应显著增强。进一步使用差分脉冲伏安法(DPV) 扫描, 发现 $\mathrm{pH}=4.0$ 时, $\mathrm{K}-\mathrm{Ti}_{3} \mathrm{C}_{2} \mathrm{~T}_{x} / \mathrm{GCE}$ 修饰电极对 $\mathrm{UO}_{2}{ }^{2+}$ 在铀浓度 $0.5 \sim 10 \mathrm{mg} / \mathrm{L}$ 范围内呈现良好的线性检测关系, 本 方法的检测限为 $0.083 \mathrm{mg} / \mathrm{L}(\mathrm{S} / \mathrm{N}=3)$, 稳定性和重复性好。

关 键 词: 铀酰离子; 碱化 $\mathrm{Ti}_{3} \mathrm{C}_{2} \mathrm{~T}_{x}$; 电化学检测

中图分类号: TQ174 文献标识码: A

\section{Alkalization Intercalation of MXene for Electrochemical Detection of Uranyl Ion}

\author{
FAN Mao ${ }^{1}$, WANG Lin ${ }^{2}$, PEI Cheng-Xin ${ }^{1}$, SHI Wei-Qun ${ }^{2}$
}

(1. State Key Laboratory of NBC Protection for Civilian, Beijing102205, China; 2. Institute of High Energy Physics, Chinese Academy of Sciences, Beijing 100049, China)

\begin{abstract}
Given the good electrochemical performance and excellent irradiation stability of two dimensional transition metal carbides (MXenes), the development of MXene-based electrode materials for radionuclide detection is very promising. In this work, $\mathrm{Ti}_{3} \mathrm{C}_{2} \mathrm{~T}_{x}$ MXene was activated via an alkalization strategy to form $\mathrm{K}^{+}$intercalated $\mathrm{Ti}_{3} \mathrm{C}_{2} \mathrm{~T}_{x}$ $\left(\mathrm{K}-\mathrm{Ti}_{3} \mathrm{C}_{2} \mathrm{~T}_{x}\right)$. Then the modified electrode of $\mathrm{K}-\mathrm{Ti}_{3} \mathrm{C}_{2} \mathrm{~T}_{x} / \mathrm{GCE}$ was prepared on glassy carbon electrode (GCE). $\mathrm{Ti}_{3} \mathrm{C}_{2} \mathrm{~T}_{x}$ and $\mathrm{K}-\mathrm{Ti}_{3} \mathrm{C}_{2} \mathrm{~T}_{x}$ were characterized by XRD, SEM and XPS techniques, and the electrochemical detection performance of $\mathrm{K}-\mathrm{Ti}_{3} \mathrm{C}_{2} \mathrm{~T}_{x} / \mathrm{GCE}$ for trace uranyl ion $\left(\mathrm{UO}_{2}{ }^{2+}\right)$ was further investigated. Cyclic voltammetry (CV) experiments showed that the electrochemical response of $\mathrm{K}-\mathrm{Ti}_{3} \mathrm{C}_{2} \mathrm{~T}_{x} / \mathrm{GCE}$ modified electrode to $\mathrm{UO}_{2}{ }^{2+}$ increased significantly. Under the differential pulse voltammetry (DPV) scanning at $\mathrm{pH} 4.0$, the $\mathrm{K}-\mathrm{Ti}_{3} \mathrm{C}_{2} \mathrm{~T}_{x} / \mathrm{GCE}$ modified electrode presented a good linear detection relationship for $\mathrm{UO}_{2}{ }^{2+}$ in the uranium concentration range of $0.5-10 \mathrm{mg} / \mathrm{L}$. The detection limit of this method is $0.083 \mathrm{mg} / \mathrm{L}(\mathrm{S} / \mathrm{N}=3)$, with decent stability and repeatability.
\end{abstract}

Key words: uranyl ion; alkalization of $\mathrm{Ti}_{3} \mathrm{C}_{2} \mathrm{~T}_{x}$; electrochemical detection

铀是一种具有长寿命放射性与高化学毒性的钣 系元素 ${ }^{[1-2]}$ 。根据所处环境不同, 铀可以呈现出二
价、三价、四价、五价、六价等多种氧化态，而 U(VI) 是其在自然界中最常见的存在形式 ${ }^{[3]}$ 。铀对于生态

收稿日期: 2018-05-17; 收到修改稿日期：2018-07-19

基金项目: 国家自然科学基金(21577144, 11675192,21790373)

National Natural Science Foundation of China (21577144, 11675192, 21790373)

作者简介: 校㯟(1994-), 男, 硕士研究生. E-mail: fanmao@ihep.ac.cn

通讯作者: 石伟群, 研究员. E-mail: shiwq@ihep.ac.cn 
圈与人类健康造成的危害主要源自于 $U(V I)$ 在环境 中的扩散, 这是由于 $\mathrm{U}(\mathrm{VI})$ 相比于其他铀的低氧化 种态水溶性和迁移能力显著增强。近年来随着我国 核能和平利用的不断发展, 产生的大量含 $\mathrm{U}(\mathrm{VI})$ 放 射性废水 ${ }^{[4]}$ 进一步增加了铀在水体中扩散风险。而 过多的铀进入人体会使肾脏结构受损, 可能导致急 性肾功能衰竭, 此外还会增加患骨癌、肝癌和血液 病的风险 ${ }^{[5-7]}$ 。因此, 监测水系统中的痕量铀是非 常必要, 对公众安全具有重要意义。

传统的铀含量检测方法主要有: 激光诱导苂光 法 $(\mathrm{LIF})^{[8]} 、 X$ 射线苂光光谱法 $(X R F)^{[9]}$ 、电感耦合等 离子体质谱法(ICP-MS $)^{[10]}$ 、原子光谱法(ETAAS $)^{[11]}$ 、 激光拉曼光谱法 ${ }^{[12]}$ 以及电感耦合等离子体光发射 光谱 $(\mathrm{ICP}-\mathrm{OES})^{[5]}$ 。这些方法往往需要昂贵的仪器和 复杂的操作, 不利于在小型实验室和现场中检测铀 酰。电化学检测具有便携性好、灵敏度高、检测仪 器简单和维护成本低等优点 ${ }^{[13]}$, 为在现场环境构建 原位监测铀酰离子的便携式设备提供了可能性。

二维材料因其优异的电、热、力学和光学性能 而受到广泛关注 ${ }^{[14-15]}$ 。2011 年, Naguib 等 ${ }^{[16]}$ 使用氢 氟酸刻蚀法首次合成了一类新型二维过渡金属碳化 物, 因其具有类似于石墨烯的层状结构而被称作 MXene。MXene 材料具有良好的导电性、亲水性和 高比表面积 ${ }^{[16-18]}$, 被广泛用于锂离子电池 ${ }^{[19]}$ 、超级 电容器 ${ }^{[20-21]}$ 等能源领域的应用研究。近期已有报 道将 MXene 用于重金属离子和 $\mathrm{H}_{2} \mathrm{O}_{2}$ 等的电化学检 测 ${ }^{[22-23]}$ 。同时, 由于 MXene 材料具有良好的耐辐照 性和热稳定性, 在放射性核素的吸附去除领域展现 了卓越的性能 ${ }^{[3,24]}$ 。基于 MXene 材料的以上优点, 本工作开展了碱化处理的 $\mathrm{Ti}_{3} \mathrm{C}_{2} \mathrm{~T}_{x}$ 对铀酰离子 $\left(\mathrm{UO}_{2}{ }^{2+}\right)$ 的电化学检测研究, 取得了初步成果。

\section{1 实验方法}

\section{$1.1 \mathrm{Ti}_{3} \mathrm{C}_{2} \mathrm{~T}_{x}$ 的制备}

将 $0.5 \mathrm{~g} \mathrm{Ti}_{3} \mathrm{AlC}_{2}$ 粉体(纯度 $98 \mathrm{wt} \%$, 北京福斯曼 科技有限公司, 实验前对原料进行了 $15 \mu \mathrm{m}$ 过篮处 理)缓慢加入 $20 \mathrm{~mL} 15 \mathrm{wt} \% \mathrm{HF}$ (稀释自 $40 \mathrm{wt} \% \mathrm{HF}$ ), 混合物在 $40^{\circ} \mathrm{C}$ 摚拌反应 $48 \mathrm{~h}$ 。将产物 $5000 \mathrm{r} / \mathrm{min}$ 离 心并水洗 6 次, 抽滤后转移至真空干燥箱, $50^{\circ} \mathrm{C}$ 真空 干燥 $12 \mathrm{~h}$, 得到多层 $\mathrm{Ti}_{3} \mathrm{C}_{2} \mathrm{~T}_{x}$ 。

\section{2 碱化 $\mathrm{K}-\mathrm{Ti}_{3} \mathrm{C}_{2} \mathrm{~T}_{\boldsymbol{x}}$ 的制备}

将 $50 \mathrm{mg} \mathrm{Ti}{ }_{3} \mathrm{C}_{2} \mathrm{~T}_{x}$ 分散在 $20 \mathrm{~mL} 1 \mathrm{~mol} / \mathrm{L}$ 的 $\mathrm{KOH}$ 溶液中, 室温下磁力摚拌 $24 \mathrm{~h}$ 。得到的悬浊液经离 心、水洗、抽滤后转移至真空干燥箱, $50^{\circ} \mathrm{C}$ 真空干燥
$12 \mathrm{~h}$, 得到 $\mathrm{K}-\mathrm{Ti}_{3} \mathrm{C}_{2} \mathrm{~T}_{\mathrm{x}}$ 。

\section{$1.3 \mathrm{~K}-\mathrm{Ti}_{3} \mathrm{C}_{2} \mathrm{~T}_{x} / \mathrm{GCE}$ 修饰电极的制备}

每次实验前, 用 1 和 $0.05 \mu \mathrm{m}$ 的氧化铝抛光粉依 次对玻碳电极 (GCE, $\phi 3 \mathrm{~mm}$ )进行打磨, 使 GCE 表 面呈现镜面。分别使用 $\mathrm{HNO}_{3}(1: 1, V / V)$ 、乙醇和水 对抛光后的 GCE 超声 $5 \mathrm{~min}$, 清洗掉电极表面的残 留杂质。取 $4 \mathrm{mg}$ 制备的碱化 $\mathrm{K}-\mathrm{Ti}_{3} \mathrm{C}_{2} \mathrm{~T}_{x}$ 固体分散在 $4 \mathrm{~mL}$ 乙醇中, 加入 $80 \mu \mathrm{L}$ Nafion 溶液( $5 \mathrm{wt} \%$ ), 超声 分散均匀。取 $4 \mu \mathrm{L}$ 的 $\mathrm{K}-\mathrm{Ti}_{3} \mathrm{C}_{2} \mathrm{~T}_{x}$ 滴涂在 GCE 上, 自 然晾干后得到修饰电极 $\mathrm{K}-\mathrm{Ti}_{3} \mathrm{C}_{2} \mathrm{~T}_{x} / \mathrm{GCE}$ 。

\section{4 表征与测试}

使用 Hitachi S-4800 场发射扫描电镜(SEM) 观测 MXene 材料碱处理前后的表面形貌。通过 Bruker D8 Advance 粉末 X 射线衍射 (XRD) 仪对 MXene 样品的特征衍射峰和晶面间距进行表征。测试条件: $\mathrm{CuK} \alpha$ 靶, $\lambda=0.15406 \mathrm{~nm}$, 步长为 $0.02^{\circ}$ 。利用 Thermo Scientific ESCALAB 250Xi 型 X 射线光电 子能谱(XPS)仪对碱化处理前后 MXene 的表面化学 组分进行测定。使用 Autolab PGSTAT 302N (Metrohm, Inc)型电化学工作站对铀酰离子的电还原信号进行 采集。

实验采用常规三电极体系进行电化学测量, 其 中 $\mathrm{K}-\mathrm{Ti}_{3} \mathrm{C}_{2} \mathrm{~T}_{x} / \mathrm{GCE}$ 修饰电极为工作电极, 铂电极为 对电极, 自制银/氯化银电极 $(\mathrm{Ag} / \mathrm{AgCl})$ 作为参比电 极。电位设置通过 NOVA 1.10 软件控制。实验在 $20 \mathrm{~mL}$ 的石英杯中进行, 以 $0.01 \mathrm{~mol} / \mathrm{L} \mathrm{NaCl}$ 作为支 持电解质, 根据需要加入适当浓度的铀酰离子溶 液, 每次测试前预调 $\mathrm{pH}$, 随后使用氩气通气 $5 \mathrm{~min}$, 去除溶液中的氧。测试过程中保持液面上方的氩气 氛围, 通过电化学工作站的循环伏安模块 $(\mathrm{CV})$ 和 差分脉冲模块(DPV)对溶液中的 $\mathrm{UO}_{2}{ }^{2+}$ 进行电化学 检测。

\section{2 结果与讨论}

\subsection{XRD 表征}

如图 1 所示, 经过 $\mathrm{HF}$ 刻蚀之后, 母体材料 $\mathrm{Ti}_{3} \mathrm{AlC}_{2}$ 在 $39.01^{\circ}$ 处的特征峰消失, 这证明了 $\mathrm{HF}$ 成 功将 $\mathrm{Ti}_{3} \mathrm{AlC}_{2}$ 刻蚀完全 ${ }^{[16]}$ 。刻蚀后 $\mathrm{Ti}_{3} \mathrm{C}_{2} \mathrm{~T}_{x}$ 的 $(002)$ 峰由 $9.56^{\circ}$ 左移至 $8.85^{\circ}$, 对应的片层材料 $c$ 轴晶格常 数 $(c$-LP)由 $1.849 \mathrm{~nm}$ 增加到 $1.997 \mathrm{~nm}$ 。经过 $\mathrm{KOH}$ 处理后的 $\mathrm{K}-\mathrm{Ti}_{3} \mathrm{C}_{2} \mathrm{~T}_{x}$ (002)峰左移至 $6.92^{\circ}, c$ - $\mathrm{LP}$ 增大 至 $2.595 \mathrm{~nm}$, 这证明碱活化处理后 $\mathrm{K}$ 离子进入到 MXene 的层间, $\mathrm{K}$ 离子的插层作用进一步扩大了 $\mathrm{Ti}_{3} \mathrm{C}_{2} \mathrm{~T}_{x}$ 的层间距。 


\subsection{SEM 形貌分析}

使用 $\mathrm{SEM}$ 观测了 $\mathrm{Ti}_{3} \mathrm{AlC}_{2}$ 以及 $\mathrm{K}$ 离子插层前后 $\mathrm{Ti}_{3} \mathrm{C}_{2} \mathrm{~T}_{x}$ 的形貌(图 2)。原始的 $\mathrm{Ti}_{3} \mathrm{AlC}_{2}$ 颗粒表现出了 紧密排列的层状结构(图 2(a))。 $\mathrm{Ti}_{3} \mathrm{AlC}_{2}$ 在经过 $48 \mathrm{~h}$ $\mathrm{HF}$ 蚀刻后, 出现了明显的分层, 得到的 $\mathrm{Ti}_{3} \mathrm{C}_{2} \mathrm{~T}_{x}$ 呈 现出手风琴状的层状结构(图 2(b)和(c))。从图 2(d) 中可以看到, 经过 $\mathrm{KOH}$ 插层活化后的 $\mathrm{K}-\mathrm{Ti}_{3} \mathrm{C}_{2} \mathrm{~T}_{x}$ 宏 观多层堆叠结构保持不变, 但其微观表面被线毛状 纳米颗粒所覆盖, 这表明在 $\mathrm{KOH}$ 的插层过程中, $\mathrm{Ti}_{3} \mathrm{C}_{2} \mathrm{~T}_{x}$ 高活性表面可能发生了部分氧化。

\subsection{XPS 分析}

使用 XPS 对 $\mathrm{K}^{+}$插层前后 $\mathrm{Ti}_{3} \mathrm{C}_{2} \mathrm{~T}_{x}$ 表面的化学成 分进行分析, 全谱扫描(图 3(a))表明 $\mathrm{Ti}_{3} \mathrm{C}_{2} \mathrm{~T}_{x}$ 样品中 含有 $\mathrm{Ti} 、 \mathrm{O} 、 \mathrm{C}$ 和 $\mathrm{F}$ 元素, 碱活化处理后在 293 和 $377 \mathrm{eV}$ 处出现了 $\mathrm{K} 2 \mathrm{p}$ 与 $\mathrm{K} 2 \mathrm{~s}$ 的特征峰, 证明了 $\mathrm{K}$ 离 子的成功插层。对 Ti2p 的高分辨 XPS 谱进行分峰 拟合, 如图 3(b)所示, 发现 454.89、455.78、456.9 和 $458.6 \mathrm{eV}$ 分别对应于 $\mathrm{Ti}-\mathrm{C} 、 \mathrm{Ti}^{2+} 、 \mathrm{Ti}^{3+} 、 \mathrm{Ti}(\mathrm{IV})-\mathrm{O}$ 的 $\mathrm{Ti} 2 \mathrm{p} 3 / 2$ 特征峰, $460 \mathrm{eV}$ 以后则为相应的 Ti2p1/2 特 征峰 ${ }^{[23]}$ 。可以看出原始 $\mathrm{Ti}_{3} \mathrm{C}_{2} \mathrm{~T}_{x}$ 样品中的 $\mathrm{Ti}$ 以低价 种态为主, 而 $\mathrm{Ti}(\mathrm{IV})-\mathrm{O}$ 的含量很少。经 $\mathrm{KOH}$ 处理

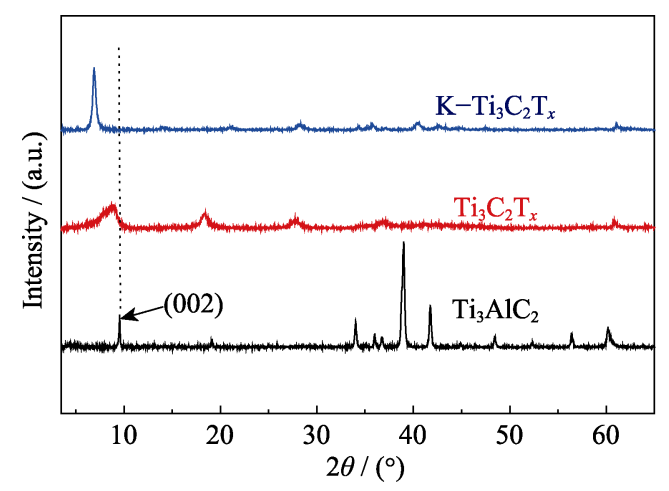

图 $1 \mathrm{Ti}_{3} \mathrm{AlC}_{2} 、 \mathrm{Ti}_{3} \mathrm{C}_{2} \mathrm{~T}_{x} 、 \mathrm{~K}-\mathrm{Ti}_{3} \mathrm{C}_{2} \mathrm{~T}_{x}$ 三种材料的 XRD 图谱 Fig. 1 XRD patterns of the synthesized $\mathrm{Ti}_{3} \mathrm{AlC}_{2} 、 \mathrm{Ti}_{3} \mathrm{C}_{2} \mathrm{~T}_{x}$ and $\mathrm{K}-\mathrm{Ti}_{3} \mathrm{C}_{2} \mathrm{~T}_{x}$
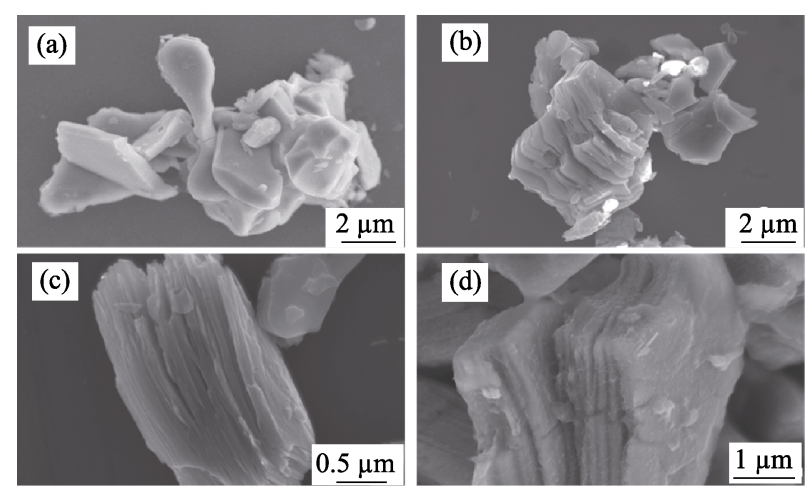

图 2 三种材料的 SEM 照片

Fig. 2 SEM images of $\mathrm{Ti}_{3} \mathrm{AlC}_{2}, \mathrm{Ti}_{3} \mathrm{C}_{2} \mathrm{~T}_{x}$ and $\mathrm{K}-\mathrm{Ti}_{3} \mathrm{C}_{2} \mathrm{~T}_{x}$ (a) $\mathrm{Ti}_{3} \mathrm{AlC}_{2}$; (b) $\mathrm{Ti}_{3} \mathrm{C}_{2} \mathrm{~T}_{x}$; (c) Enlarged view of $\mathrm{Ti}_{3} \mathrm{C}_{2} \mathrm{~T}_{x}$; (d) $\mathrm{K}-\mathrm{Ti}_{3} \mathrm{C}_{2} \mathrm{~T}_{x}$
后 $\mathrm{K}-\mathrm{Ti}_{3} \mathrm{C}_{2} \mathrm{~T}_{x}$ 中低价 $\mathrm{Ti}$ 种态减少, $\mathrm{Ti}(\mathrm{IV})-\mathrm{O}$ 特征峰所 占比例显著增加, 并且其峰位与 $\mathrm{TiO}_{2}$ 的 $\mathrm{Ti}-\mathrm{O}$ 键结合 能数值非常接近 ${ }^{[24]}$ 。而 $\mathrm{O} 1 \mathrm{~s}$ 高分辨 XPS 图谱(图 3(c) 也表明碱活化后样品中的氧含量有所增加。以上结 果确认了 $\mathrm{KOH}$ 处理使得 $\mathrm{Ti}_{3} \mathrm{C}_{2} \mathrm{~T}_{x}$ 样品表面部分氧化, 结合之前的 SEM 结果, 可推知 $\mathrm{K}-\mathrm{Ti}_{3} \mathrm{C}_{2} \mathrm{~T}_{x}$ 表面形成 了少量 $\mathrm{TiO}_{2}$ 纳米颗粒 ${ }^{[25]}$ 。

\section{$2.4 \mathrm{UO}_{2}{ }^{2+}$ 在修饰电极上的循环伏安行为}

\subsection{1 电化学响应增益}

分别使用 $\mathrm{GCE} 、 \mathrm{Ti}_{3} \mathrm{C}_{2} \mathrm{~T}_{x}$ 修饰的玻碳电极 $\left(\mathrm{Ti}_{3} \mathrm{C}_{2} \mathrm{~T}_{x} / \mathrm{GCE}\right)$ 以及 $\mathrm{K}-\mathrm{Ti}_{3} \mathrm{C}_{2} \mathrm{~T}_{x} / \mathrm{GCE}$ 在 $\mathrm{pH}=4$, $-0.6 \sim-0.1 \mathrm{~V}$ 的电位范围内, 对 $50 \mathrm{mg} / \mathrm{L}$ 的 $\mathrm{U}(\mathrm{VI})$ 进 行循环伏安扫描, 扫速为 $100 \mathrm{mV} / \mathrm{s}$, 每组进行 10 次
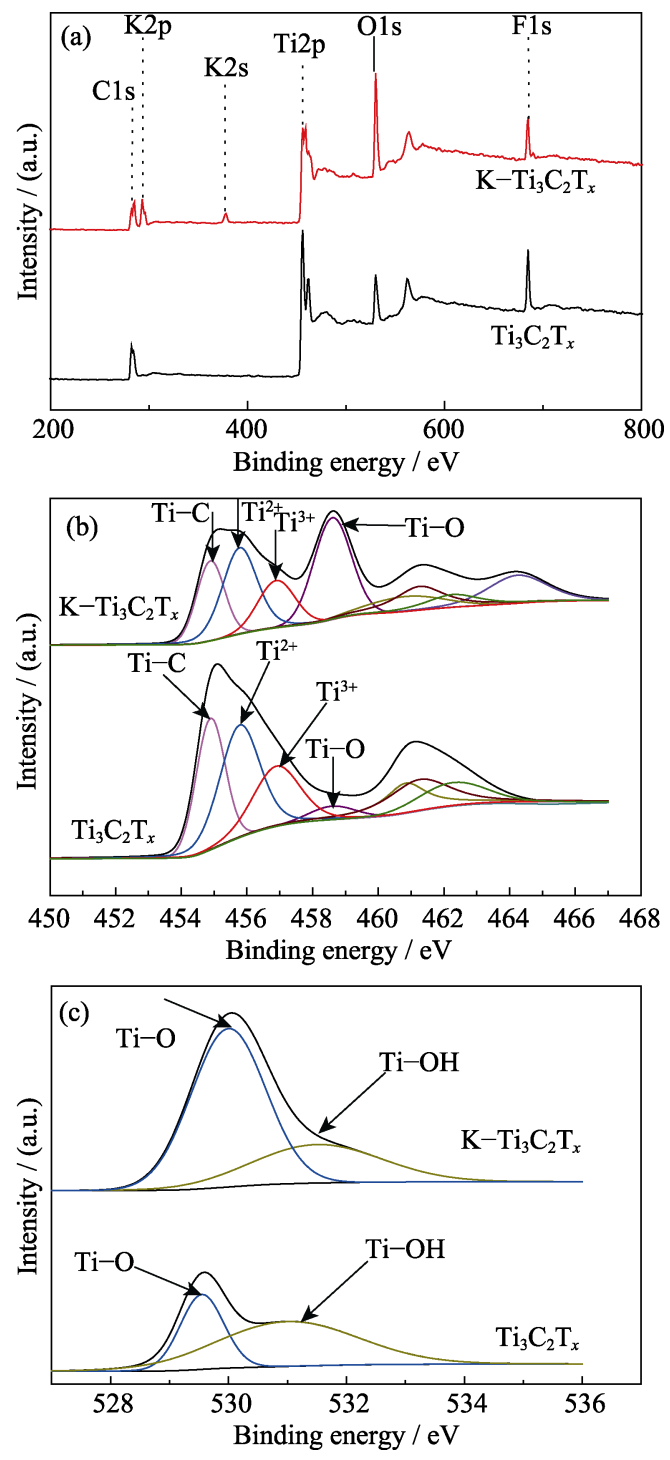

图 3 (a) $\mathrm{Ti}_{3} \mathrm{C}_{2} \mathrm{~T}_{x}$ 和 $\mathrm{K}-\mathrm{Ti}_{3} \mathrm{C}_{2} \mathrm{~T}_{x}$ 的 XPS 光谱图, (b) $\mathrm{Ti}_{3} \mathrm{C}_{2} \mathrm{~T}_{x}$ 和 $\mathrm{K}-\mathrm{Ti}_{3} \mathrm{C}_{2} \mathrm{~T}_{x}$ 的 $\mathrm{Ti} 2 \mathrm{p}$ 高分辨图, (c) $\mathrm{Ti}_{3} \mathrm{C}_{2} \mathrm{~T}_{x}$ 和 $\mathrm{K}-\mathrm{Ti}_{3} \mathrm{C}_{2} \mathrm{~T}_{x}$ 的 $\mathrm{O} 1 \mathrm{~s}$ 高分辨图

Fig. 3 (a) XPS spectra of $\mathrm{Ti}_{3} \mathrm{C}_{2} \mathrm{~T}_{x}$ and $\mathrm{K}-\mathrm{Ti}_{3} \mathrm{C}_{2} \mathrm{~T}_{x}$, (b) highresolution Ti2p spectra of MXene $\mathrm{Ti}_{3} \mathrm{C}_{2} \mathrm{~T}_{x}$ and $\mathrm{K}-\mathrm{Ti}_{3} \mathrm{C}_{2} \mathrm{~T}_{x}$, (c) high- resolution O1s spectra of $\mathrm{MXene} \mathrm{Ti}_{3} \mathrm{C}_{2} \mathrm{~T}_{x}$ and $\mathrm{K}-\mathrm{Ti}_{3} \mathrm{C}_{2} \mathrm{~T}_{x}$ 
平行扫描(图 4 所示)。玻碳电极上 $\mathrm{UO}_{2}{ }^{2+}$ 在 $-0.35 \mathrm{~V}$ 的电位出现微弱的还原峰信号 (图 4(A)中(b)所示),

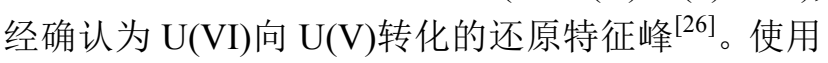
硝酸钠代替硝酸铀酰进行空白实验，在循环伏安扫 描范围内未检出氧化还原峰, 表明阴离子对于铀酰 离子的检测无显著影响。 $\mathrm{Ti}_{3} \mathrm{C}_{2} \mathrm{~T}_{x} / \mathrm{GCE}$ 修饰电极由 于多层 $\mathrm{Ti}_{3} \mathrm{C}_{2} \mathrm{~T}_{x}$ 的电容效应 ${ }^{[20]}$, 遮盖了电极对 $\mathrm{UO}_{2}{ }^{2+}$ 的还原峰信号(图 4(B)所示)。而 $\mathrm{K}-\mathrm{Ti}_{3} \mathrm{C}_{2} \mathrm{~T}_{x} / \mathrm{GCE}$ 修 饰电极相较于纯玻碳电极, 出现了更强的还原峰 (图 4(A)中(c)所示)。 $\mathrm{K}-\mathrm{Ti}_{3} \mathrm{C}_{2} \mathrm{~T}_{x} / \mathrm{GCE}$ 对 U(VI) 具有更 高的电化学响应主要原因如下: (1)相比于 GCE, $\mathrm{K}-\mathrm{Ti}_{3} \mathrm{C}_{2} \mathrm{~T}_{x}$ 自身的多层结构使其具有更大的比表面积以 及更多的吸附位点; (2)K- $\mathrm{Ti}_{3} \mathrm{C}_{2} \mathrm{~T}_{\mathrm{x}}$ 表面带有负电荷 ${ }^{[27]}$, 有利于 $\mathrm{UO}_{2}{ }^{2+}$ 阳离子的预吸附富集; (3)K 离子的插 层提高了 $\mathrm{Ti}_{3} \mathrm{C}_{2} \mathrm{~T}_{x}$ 的层间稳定性 ${ }^{[28]}$, 从而有效抑制 循环伏安扫描时电解质进出 MXene 层间产生的电容 效应; (4)已有研究表明 Ti-O 键对重金属离子以及钣 系离子具有较强的配位作用 ${ }^{[27,29]}$, 碱活化处理使 MXene 表面的 Ti-O 和 Ti-OH 端基含量增加(图 3(c)), 并且形成了部分的纳米 $\mathrm{TiO}_{2}{ }^{[25]}$, 提供了更多的 $\mathrm{UO}_{2}{ }^{2+}$ 活性配位位点, 从而提高了电化学响应的选择性。

\subsection{2 $\mathrm{pH}$ 的影响}

将修饰电极在 $0.01 \mathrm{~mol} / \mathrm{L}$ 的 $\mathrm{NaCl}$ 溶液中, 用
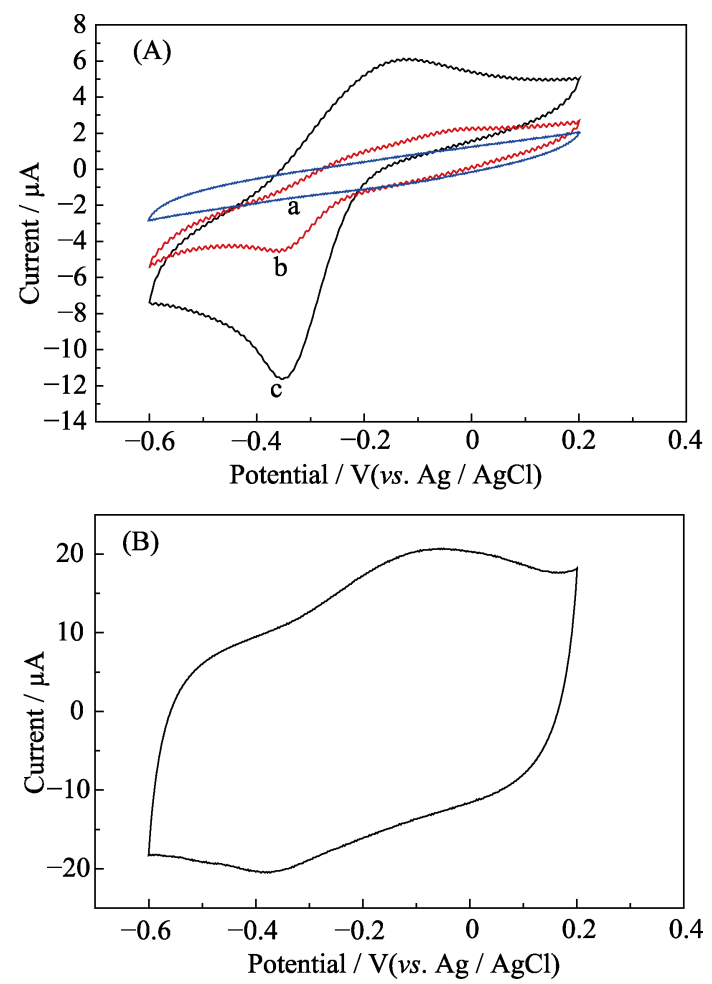

图 $4 \mathrm{UO}_{2}{ }^{2+}$ 在不同电极上的循环伏安 $(\mathrm{CV})$ 响应

Fig. 4 Cyclic voltammetry $(\mathrm{CV})$ results of $\mathrm{UO}_{2}{ }^{2+}$ in different electrodes

(A) (a) $\mathrm{K}-\mathrm{Ti}_{3} \mathrm{C}_{2} \mathrm{~T}_{x} / \mathrm{GCE},[\mathrm{U}]=0 \mathrm{mg} / \mathrm{L}$, (b) GCE, $[\mathrm{U}]=50 \mathrm{mg} / \mathrm{L}$, (c) $\mathrm{K}$ $\mathrm{Ti}_{3} \mathrm{C}_{2} \mathrm{~T}_{x} / \mathrm{GCE},[\mathrm{U}]=50 \mathrm{mg} / \mathrm{L}$; (B) $\mathrm{Ti}_{3} \mathrm{C}_{2} \mathrm{~T}_{x} / \mathrm{GCE},[\mathrm{U}]=50 \mathrm{mg} / \mathrm{L}$
$50 \mathrm{mV} / \mathrm{s}$ 的扫速, 分别在 $\mathrm{pH}$ 为 $3 、 4$ 和 5 的条件下, 对 $50 \mathrm{mg} / \mathrm{L}$ 的 $\mathrm{U}(\mathrm{VI})$, 在 $-0.6 \sim 0.2 \mathrm{~V}$ 电位区间内进行循 环伏安 $(\mathrm{CV})$ 扫描。如图 5 结果显示, 在 $\mathrm{pH}$ 为 4 的条 件下, 修饰电极 $\mathrm{K}-\mathrm{Ti}_{3} \mathrm{C}_{2} \mathrm{~T}_{x} / \mathrm{GCE}$ 对 $\mathrm{UO}_{2}{ }^{2+}$ 具有较好的 响应, 峰电流大, 还原峰明显。当 $\mathrm{pH}$ 升高到 5 时, 还 原峰电流降低, 这可能与高 $\mathrm{pH}$ 下溶液中部分铀酰 离子以 $\left(\mathrm{UO}_{2}\right)_{2}(\mathrm{OH})_{2}{ }^{2+} 、\left(\mathrm{UO}_{2}\right)_{3}(\mathrm{OH})_{5}{ }^{+}$等多核种态的形 式存在, 不利于 $U(V I)$ 的电化学还原有关。当 $\mathrm{pH}$ 降 低到 3 时, 背景电流增大使得还原峰的峰型变得不 再尖锐, 不利于 $\mathrm{U}(\mathrm{VI})$ 还原峰的检测。由于 $\mathrm{H}^{+}$的离 子半径很小, 更易在 MXene 层间扩散迁移, 低 $\mathrm{pH}$ 下溶液中 $\mathrm{H}^{+}$浓度显著增加, 从而导致循环伏安扫描 所引起的电容效应背景电流增大。因此, 选择 $\mathrm{pH}=4$ 作为 $\mathrm{UO}_{2}{ }^{2+}$ 检测的最佳 $\mathrm{pH}$ 。

\subsection{3 不同扫速下循环伏安图}

修饰电极在 $0.01 \mathrm{~mol} / \mathrm{LNaCl}, \mathrm{pH}=4.0$, 在 -0.6 $0.2 \mathrm{~V}$ 电位区间, 以不同扫速对 $50 \mathrm{mg} / \mathrm{L}$ 的 U(VI)进 行循环伏安扫描。如图 6(a)所示, 在 0.01 0.1 V/s 的 范围内, 氧化峰和还原峰的大小均与扫速的平方根 成正比, 如图 6(b)所示, 其相关系数分别为 0.997 和 0.999 , 说明此时的电极过程主要受扩散控制, 电极 表面发生氧化还原反应。

\section{5 差分脉冲伏安(DPV)对 U(VI)的测定}

\subsubsection{U(VI)标准曲线}

基于以上的测试结果, 本课题组在 $\mathrm{pH}$ 为 4 , 电 解质为 $0.01 \mathrm{~mol} / \mathrm{L}$ 的 $\mathrm{NaCl}$ 中, 在 $-0.6 \sim-0.1 \mathrm{~V}$ 的电 位范围, 使用差分脉冲伏安法(DPV)对 $\mathrm{UO}_{2}{ }^{2+}$ 的还原 峰进行电化学检测。结果显示, 在 $-0.35 \mathrm{~V}$ 左右的 电位时, 出现 $\mathrm{UO}_{2}{ }^{2+}$ 的还原峰, 这与循环伏安 $(\mathrm{CV})$ 的结果相吻合。进一步研究发现, 在 $0.5 \sim 10 \mathrm{mg} / \mathrm{L}$ 铀 浓度范围内, 修饰电极对 $\mathrm{U}(\mathrm{VI})$ 呈现良好的线性关 系, 标准曲线为 $y(\mu \mathrm{A})=0.4356 x(\mathrm{mg} / \mathrm{L})+3.7375$, 相 关系数 $R^{2}=0.997$ (图 7 所示)。对同一空白对照溶液 平行测量 10 次, 计算空白溶液的标准偏差, 并以 3

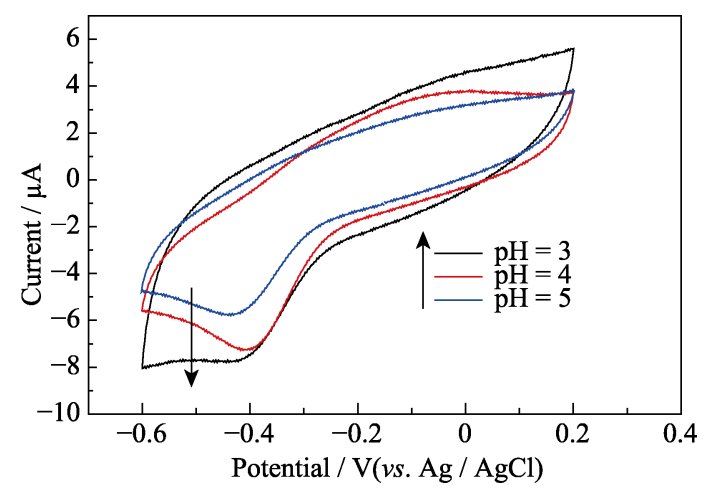

图 $5 \mathrm{pH}$ 对 $\mathrm{K}-\mathrm{Ti}_{3} \mathrm{C}_{2} \mathrm{~T}_{\mathrm{x}} / \mathrm{GCE}$ 检测 $\mathrm{UO}_{2}{ }^{2+}$ 的影响

Fig. 5 The effect of $\mathrm{pH}$ on $\mathrm{UO}_{2}{ }^{2+} /\left(\mathrm{K}-\mathrm{Ti}_{3} \mathrm{C}_{2} \mathrm{~T}_{x} / \mathrm{GCE}\right)$ 

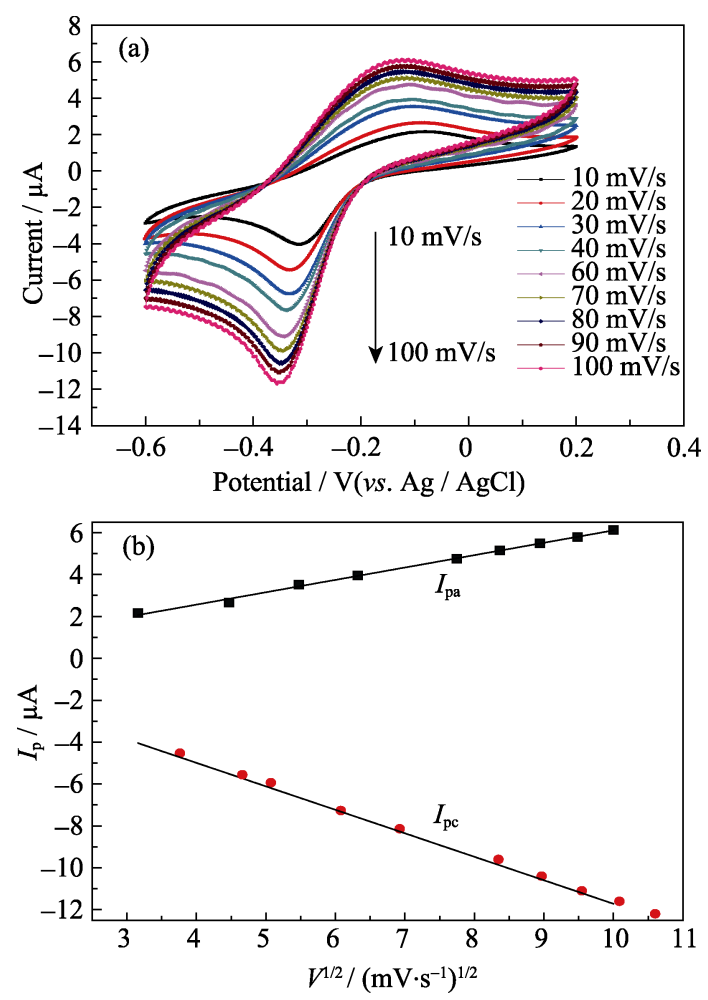

图 $6 \mathrm{~K}-\mathrm{Ti}_{3} \mathrm{C}_{2} \mathrm{~T}_{x} \mathrm{GCE}$ 于 $0.01 \mathrm{~mol} / \mathrm{L} \mathrm{pH}=4.0$ 的 $\mathrm{NaCl}$ 中对 $\mathrm{UO}_{2}{ }^{2+}$ 的循环伏安扫描结果, 扫描速度为 $10 \sim 100 \mathrm{mV} / \mathrm{s}$, [U] $=50 \mathrm{mg} / \mathrm{L}$

Fig. 6 Cyclic voltammetry results of $\mathrm{UO}_{2}{ }^{2+} /\left(\mathrm{K}_{-}-\mathrm{Ti}_{3} \mathrm{C}_{2} \mathrm{~T}_{x} /\right.$ GCE), $\mathrm{pH} 4.0,10-100 \mathrm{mV} / \mathrm{s},[\mathrm{U}]=50 \mathrm{mg} / \mathrm{L}$

(a) CV curves; (b) Plots of peak currents $v s$ scan speed
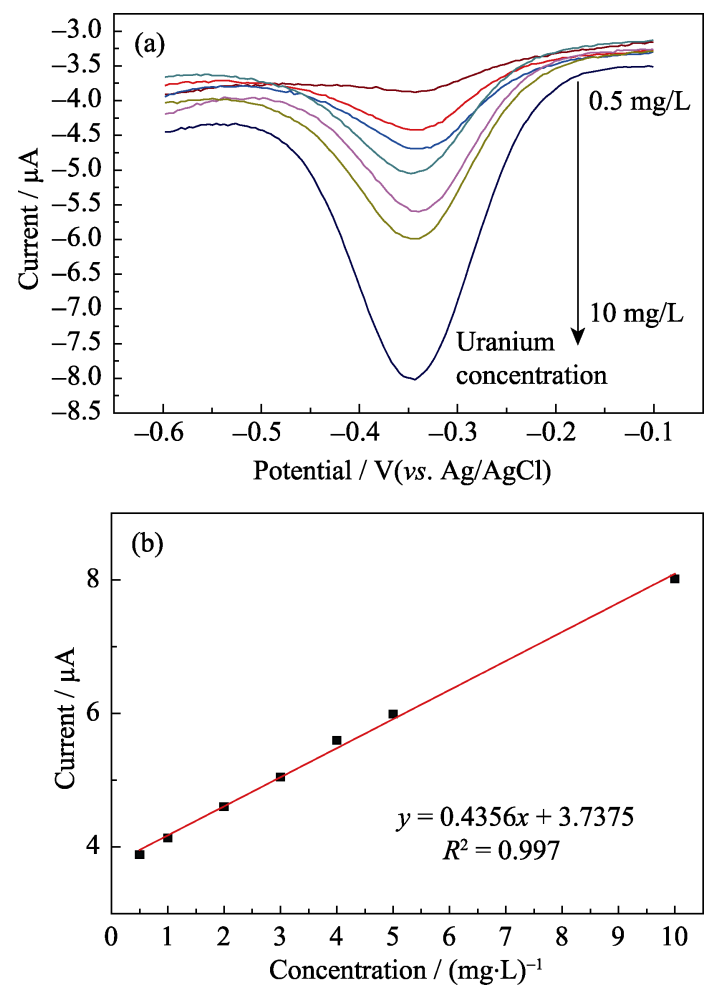

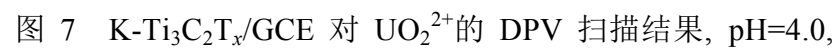
$[\mathrm{U}]=0.5 \sim 10 \mathrm{mg} / \mathrm{L}$

Fig 7 Differential pulse voltammetry (DPV) results of $\mathrm{UO}_{2}{ }^{2+}$ / $\left(\mathrm{K}^{-} \mathrm{Ti}_{3} \mathrm{C}_{2} \mathrm{~T}_{x} / \mathrm{GCE}\right), \mathrm{pH}=4.0,[\mathrm{U}]=0.5-10 \mathrm{mg} / \mathrm{L}$

(a) DPV curves; (b) Plots of peak currents $v s$ concentration of uranium
倍空白溶液的标准偏差作为检出限, 计算方法的检 测限为 $0.089 \mathrm{mg} / \mathrm{L}(\mathrm{S} / \mathrm{N}=3)$ 。

\subsection{2 实际样品测试}

选取实验室中的自来水作为空白水体，加 $0.01 \mathrm{~mol} / \mathrm{L}$ 氯化钠作为支持电解液, 加入 $5 \mathrm{mg} / \mathrm{L}$ 的 $\mathrm{U}(\mathrm{VI})$, 进行实际测试。用上述测试方法中的差分脉 冲伏安法(DPV)进行测试分析, 记录还原峰电流, 通过标准曲线得到测量的水体中铀的浓度。对样品 平行测试 3 次, 自来水中样品的测试结果如下表 1 。 自来水中铀浓度的测试结果为 $5.30 \mathrm{mg} / \mathrm{L}$, 相对标 准方差为 $1.32 \%$, 表明测试方法的准确性和稳定性 好，可以应用于实际水体中痕量铀的定量检测。

\subsection{3 回收率、稳定性和重现性}

以 $1 \mathrm{mg} / \mathrm{L}$ 的 U(VI)溶液作为底液, 按上述样品 分析步骤进行回收率测试, 测试结果列于表 2 。四次 加标回收率在 $93 \% \sim 103 \%$ 之间，同时每个浓度三个 平行测试得到的 $\mathrm{RSD}$ 小于 $2 \%$, 表明 $\mathrm{K}-\mathrm{Ti}_{3} \mathrm{C}_{2} \mathrm{~T}_{x}$ 电极 对 U(VI)的检测结果具有良好的可靠性。用修饰电 极对 $5 \mathrm{mg} / \mathrm{L}$ 的 U(VI)溶液进行连续 10 次的差分脉 冲伏安法扫描, 十次测定的还原峰的电流的相对标 准偏差为 $1.9 \%$, 说明测试过程的电流响应重现性良 好。将同一批次的 $\mathrm{K}-\mathrm{Ti}_{3} \mathrm{C}_{2} \mathrm{~T}_{x}$ 材料在 $4^{\circ} \mathrm{C}$ 下保存, 放 置一个月后, 制备的修饰电极 $\mathrm{K}-\mathrm{Ti}_{3} \mathrm{C}_{2} \mathrm{~T}_{x} / \mathrm{GCE}$ 对 $5 \mathrm{mg} / \mathrm{L}$ 的 U(VI)使用差分脉冲伏安法 DPV 进行扫描, 结果显示 $\mathrm{UO}_{2}{ }^{2+}$ 的还原峰电流保持在 $95 \%$ 以上, 说 明材料具有很好的稳定性。

表 1 自来水中样品的分析结果

Table 1 Sample analysis results of uranium content in tap-water

\begin{tabular}{cccccc}
\hline Samples & number & $\begin{array}{c}\text { Detected } \\
/\left(\mathrm{mg} \cdot \mathrm{L}^{-1}\right)\end{array}$ & $\begin{array}{c}\text { Found } \\
/\left(\mathrm{mg} \cdot \mathrm{L}^{-1}\right)\end{array}$ & $\begin{array}{c}\text { Average } \\
/\left(\mathrm{mg} \cdot \mathrm{L}^{-1}\right)\end{array}$ & $\mathrm{RSD} / \%$ \\
\hline \multirow{3}{*}{ Tap-water } & 2 & 5.00 & 5.36 & & \\
& 3 & 5.00 & 5.22 & 5.30 & 1.34 \\
& 3.00 & 5.31 & & \\
\hline
\end{tabular}

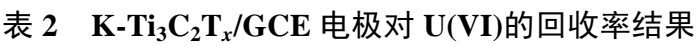
Table 2 Determination of U(VI) in the recovery experiments

\begin{tabular}{cccccc}
\hline Samples & $\begin{array}{c}\text { Detected } \\
/\left(\mathrm{mg} \cdot \mathrm{L}^{-1}\right)\end{array}$ & $\begin{array}{c}\text { Added } \\
/\left(\mathrm{mg} \cdot \mathrm{L}^{-1}\right)\end{array}$ & $\begin{array}{c}\text { Found } \\
/\left(\mathrm{mg} \cdot \mathrm{L}^{-1}\right)\end{array}$ & $\begin{array}{c}\text { Recovery } \\
/ \%\end{array}$ & $\mathrm{RSD} / \%$ \\
\hline 1 & 1.0 & 1.0 & 1.93 & 93.00 & 1.95 \\
2 & 1.0 & 2.0 & 3.06 & 103.00 & 1.54 \\
3 & 1.0 & 3.0 & 3.95 & 98.00 & 1.12 \\
4 & 1.0 & 4.0 & 5.11 & 102.75 & 1.23 \\
\hline
\end{tabular}




\section{3 结论}

使用 $\mathrm{HF}$ 刻蚀法和碱化插层后处理的方法, 分 别得到了 $\mathrm{Ti}_{3} \mathrm{C}_{2} \mathrm{~T}_{x}$ 和钾离子插层的 $\mathrm{K}-\mathrm{Ti}_{3} \mathrm{C}_{2} \mathrm{~T}_{x}$ 两种二 维层状 MXene 材料。循环伏安扫描结果显示, $\mathrm{K}-\mathrm{Ti}_{3} \mathrm{C}_{2} \mathrm{~T}_{x}$ 对玻碳电极的修饰可以显著提高对 $\mathrm{UO}_{2}{ }^{2+}$ 的电化学响应, 这是由于 $\mathrm{Ti}_{3} \mathrm{C}_{2} \mathrm{~T}_{x}$ 本身具有良好的 导电性、较大的比表面积以及负的表面电荷。同时 碱化过程使得 $\mathrm{Ti}_{3} \mathrm{C}_{2} \mathrm{~T}_{x}$ 表面 Ti-O/Ti-OH 端基含量增 加, 并且生成了部分 $\mathrm{TiO}_{2}$ 纳米颗粒, 从而进一步增 加 $\mathrm{U}(\mathrm{VI})$ 活性配位位点。此外, $\mathrm{K}$ 离子在 $\mathrm{Ti}_{3} \mathrm{C}_{2} \mathrm{~T}_{x}$ 层 间的插层消除了 $\mathrm{Ti}_{3} \mathrm{C}_{2} \mathrm{~T}_{x}$ 自身电容效应的干扰。DPV 结果显示, $\mathrm{K}_{-}-\mathrm{Ti}_{3} \mathrm{C}_{2} \mathrm{~T}_{x} / \mathrm{GCE}$ 修饰电极在铀含量 $0.5 \mathrm{mg} / \mathrm{L}$ 至 $10 \mathrm{mg} / \mathrm{L}$ 范围内, 对 $\mathrm{UO}_{2}{ }^{2+}$ 的还原峰和峰电流呈现 良好的线性关系，为 $y(\mathrm{~A})=0.4356 x(\mathrm{mg} / \mathrm{L})+3.7375$, 相关系数 $R^{2}=0.997$, 检测限为 $0.083 \mathrm{mg} / \mathrm{L}(\mathrm{S} / \mathrm{N}=3)$, 可靠性、稳定性和重复性好, 有望应用于水体中痕 量铀的定量检测。

\section{参考文献:}

[1] LI J, WANG X, ZHAO G, et al. Metal-organic framework-based materials: superior adsorbents for the capture of toxic and radioactive metal ions. Chemical Society Reviews, 2018, 47(7): 2322-2356.

[2] HONGWEI P, XIANGXUE W, WEN Y, et al. Removal of radionuclides by metal oxide materials and mechanism research. Scientia Sinica Chimica, 2017, 48(1): 58-73.

[3] SANTOS J S, TEIXEIRA L S G, DOS SANTOS W N L, et al. Uranium determination using atomic spectrometric techniques: an overview. Analytica Chimica Acta, 2010, 674(2): 143-156.

[4] WANG L, YUAN L, CHEN K, et al. Loading actinides in multilayered structures for nuclear waste treatment: the first case study of uranium capture with vanadium carbide MXene. ACS Applied Materials \& Interfaces, 2016, 8(25): 16396-16403.

[5] JAMALI M R, ASSADI Y, SHEMIRANI F, et al. Synthesis of salicylaldehyde-modified mesoporous silica and its application as a new sorbent for separation, preconcentration and determination of uranium by inductively coupled plasma atomic emission spectrometry. Analytica Chimica Acta, 2006, 579(1): 68-73.

[6] MEHRA R, SINGH S, SINGH K. Uranium studies in water samples belonging to Malwa region of Punjab, using track etching technique. Radiation Measurements, 2007, 42(3): 441-445.

[7] ZIKOVSKY L. Determination of uranium in food in Quebec by neutron activation analysis. Journal of Radioanalytical and $\mathrm{Nu}$ clear Chemistry, 2006, 267(3): 695-697.

[8] KUMAR S A, SHENOY N S, PANDEY S, et al. Direct determination of uranium in seawater by laser fluorimetry. Talanta, 2008, 77(1): 422-426.

[9] MISRA N L, DHARA S, MUDHER K D S. Uranium determination in seawater by total reflection X-ray fluorescence spectrometry. Spectrochimica Acta Part B: Atomic Spectroscopy, 2006, 61(10/11): 1166-1169.

[10] AVIVAR J, FERRER L, CASAS M, et al. Fully automated lab-on-valve-multisyringe flow injection analysis-ICP-MS system: an effective tool for fast, sensitive and selective determination of thorium and uranium at environmental levels exploiting solid phase extraction. Journal of Analytical Atomic Spectrometry, 2012,
27(2): 327-334.

[11] KUMAR M, RATHORE D P S, SINGH A K. Pyrogallol immobilized Amberlite XAD-2: a newly designed collector for enrichment of metal ions prior to their determination by flame atomic absorption spectrometry. Microchimica Acta, 2001, 137(3/4): 127-134.

[12] CHO H R, JUNG E C, CHA W, et al. Quantitative analysis of uranium in aqueous solutions using a semiconductor laser-based spectroscopic method. Analytical Chemistry, 2013, 85(9): 4279-4283.

[13] ZHANG L, WANG C Z, TANG H B, et al. Rapid determination of uranium in water samples by adsorptive cathodic stripping voltammetry using a tin-bismuth alloy electrode. Electrochimica Acta, 2015, 174: 925-932.

[14] AHMED S, YI J. Two-dimensional transition metal dichalcogenides and their charge carrier mobilities in field-effect transistors. Nano-Micro Letters, 2017, 9(4): 50-1-23.

[15] XIAO J, ZHANG Y, CHEN H, et al. Enhanced performance of a monolayer $\mathrm{MoS}_{2} / \mathrm{WSe}_{2}$ heterojunction as a photoelectrochemical cathode. Nano-Micro Letters, 2018, 10(4): 60-1-9.

[16] NAGUIB M, KURTOGLU M, PRESSER V, et al. Two-dimensional nanocrystals produced by exfoliation of $\mathrm{Ti}_{3} \mathrm{AlC}_{2}$. Advanced Materials, 2011, 23(37): 4248-4253.

[17] LUKATSKAYA M R, MASHTALIR O, REN C E, et al. Cation intercalation and high volumetric capacitance of two-dimensional titanium carbide. Science, 2013, 341(6153): 1502-1505.

[18] MASHTALIR O, NAGUIB M, MOCHALIN V N, et al. Intercalation and delamination of layered carbides and carbonitrides. Nature Communications, 2013, 4: 1716-1-7.

[19] ZHOU J, ZHA X, ZHOU X, et al. Synthesis and electrochemical properties of two-dimensional hafnium carbide. ACS Nano, 2017, 11(4): 3841-3850.

[20] LI H, HOU Y, WANG F, et al. Flexible all-solid-state supercapacitors with high volumetric capacitances boosted by solution processable MXene and electrochemically exfoliated graphene. Advanced Energy Materials, 2017, 7(4): 1601847-1-6.

[21] LUO J, ZHANG W, YUAN H, et al. Pillared structure design of MXene with ultralarge interlayer spacing for high-performance lithium-ion capacitors. ACS Nano, 2017, 11(3): 2459-2469.

[22] LORENCOVÁ L, BERTOK T, DOSEKOVA E, et al. Electrochemical performance of $\mathrm{Ti}_{3} \mathrm{C}_{2} \mathrm{~T}_{x}$ MXene in aqueous media: towards ultrasensitive $\mathrm{H}_{2} \mathrm{O}_{2}$ sensing. Electrochimica Acta, 2017, 235: 471-479.

[23] ZHU X, LIU B, HOU H, et al. Alkaline intercalation of $\mathrm{Ti}_{3} \mathrm{C}_{2}$ MXene for simultaneous electrochemical detection of $\mathrm{Cd}$ (II), $\mathrm{Pb}$ (II), $\mathrm{Cu}$ (II) and $\mathrm{Hg}$ (II). Electrochimica Acta, 2017, 248: 46-57.

[24] BONATO M, ALLEN G C, SCOTT T B. Reduction of U(VI) to $\mathrm{U}(\mathrm{IV})$ on the surface of $\mathrm{TiO}_{2}$ anatase nanotubes. IET Micro \& Nano Letters, 2008, 3(2): 57-61.

[25] LIU Y, DU H, ZHANG X, et al. Superior catalytic activity derived from a two-dimensional $\mathrm{Ti}_{3} \mathrm{C}_{2}$ precursor towards the hydrogen storage reaction of magnesium hydride. Chemical Communications, 2016, 52(4): 705-708.

[26] KANETO K, BIDAN G. Electrochemical recognition and immobilization of uranyl ions by polypyrrole film doped with calix [6] arene. Thin Solid Films, 1998, 331(1/2): 272-278.

[27] WANG L, TAO W, YUAN L, et al. Rational control of the interlayer space inside two-dimensional titanium carbides for highly efficient uranium removal and imprisonment. Chemical Communications, 2017, 53(89): 12084-12087.

[28] OSTI N C, NAGUIB M, OSTADHOSSEIN A, et al. Effect of metal ion intercalation on the structure of MXene and water dynamics on its internal surfaces. ACS Applied Materials \& Interfaces, 2016, 8(14): 8859-8863.

[29] PENG Q, GUO J, ZHANG Q, et al. Unique lead adsorption behavior of activated hydroxyl group in two-dimensional titanium carbide. Journal of the American Chemical Society, 2014, 136(11): 4113-4116. 